\title{
MODIFICATIONS OF A SIMPLE I-BEAM AND ITS EFFECTS ON THE STRESS STATE
}

\author{
Jakub CEJPEK ${ }^{1}$, Jaroslav JURAČKA ${ }^{2}$ \\ Institute of Aerospace Engineering, Brno, Czech Republic \\ E-mail: cejpek@seznam.cz (corresponding author); juracka@fme.vutbr.cz
}

Received 22 September 2014; accepted 6 June 2016

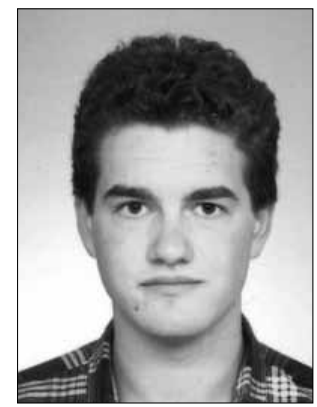

Jakub CEJPEK, Eng.

Date of birth: 1987.

Education: Eng. degree in Aeronautics, Brno University of Technology, Faculty of

Mechanical Engineering, 2009 to 2011.

Affiliations: since 2011, Researcher, Brno University of Technology, Institute of Aeronautical Engineering.

Research interest: composite structures with an emphasis on thick laminates.

Publications: author of 2 conference papers.

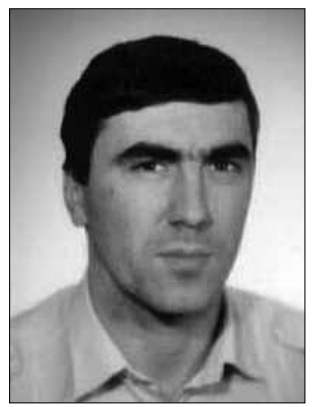

Jaroslav JURAČKA, Assoc. Prof. Eng. PhD.

Education: Eng. degree in Aeronautics, Brno University of Technology, Faculty of

Mechanical Engineering in 1994.

PhD degree in Composite Materials, Brno University of Technology, Faculty of Mechanical Engineering in 2001.

Assoc. Prof. degree, Brno University of Technology, Faculty of Mechanical Engineering in 2005.

Affiliations and functions: 2000 to 2006, Laboratory director, Brno University of

Technology, Institute of Aeronautical Engineering. Since 2005, Assoc. Prof. at Brno

University of Technology, Institute of Aeronautical Engineering. Since 2012, Director of the

Institute of Aeronautical Engineering at Brno University of Technology.

Research interests: composite materials, airplane airworthiness and certification.

Publications: author or co-author of 20 articles and many conference presentations.

\begin{abstract}
The motivation for this work is a desire for a deeper understanding of the structural failures in a composite glider wing, which has been tested in the laboratories of the Institute of Aerospace Engineering, Faculty of Mechanical Engineering, Brno University of Technology. To understand the causes of the encountered failures, one has to consider the effects of all the stages in the design, manufacturing and testing of the wing. This paper focuses only on the design stage. The presented facts were obtained from a finite element analysis. The geometry used for the analysis is that of the tested specimens. This allows validating the results by the comparison of the deformation and strains measured during the laboratory tests. The analysis starts with a simple I-beam loaded by three-point-bending. In the next step a cantilever is added. Several more modifications follow, changing the I-beam to the wing. The case evaluation considers the interaction between normal (material direction 1) and inter-laminar shear stresses in the upper flange. The goal of this paper is to quantify the effect of each design change in the wing structure and loading on the stress plane $\sigma 1-\tau 31$.
\end{abstract}

Keywords: beam, normal stress, shear stress, classical laminate theory, wing, composite.

\section{Introduction}

The wing is the most recognizable and most interesting part of the airplane. From the design and structural point of view it seems very simple: it consists of only three different basic structural parts (beam, ribs and skin); there are no complicated mechanisms; and, most often, it is a straight and linear geometry.

In the last 110 years of aviation history and design process, a long-proven beam-theory has been used. By means of simplifications and assumptions, one can 
calculate the stresses in the wing and achieve a good product, which is light, yet strong enough.

The classical beam theory was created assuming a homogenous isotropic material - like steel or aluminium alloy. However, since the middle of the 20th century, a new kind of material suitable for airplane manufacturing became available on the market. This material is a composite - a combination of two different constituents. One is the fibre - giving the composite its strength. The other is the bounding agent - glue, which keeps the fibres in the right position.

Composites spread fast and many designers have found the benefits of the light-weight, sleek surface, strength, shape adjustability and good fatigue properties.

Designers and engineers used to isotropic metals had to deal with the composite anisotrophy. At first, the directional properties' difference led to a new failure index definition. Later, the ply-nature of the composites had to be described. Thus, out of the Midlins Plate theory, the classical laminate theory has been developed (Liu 2003).

Tools dedicated to composite design evolved over the years. Most of composite design is done on a computer nowadays. However, even today, most of the computer programs still use the same assumptions about thin plates and zero through-thickness stresses. Such assumptions are not always correct and deeper analysis is required.

When discussing small airplanes, one or two seat gliders and ultralight sport airplanes, one has to keep in mind the limited possibilities of the designers in terms of technological support (material and strength tests), equipment (expensive computers and software), and, last but not least, the time pressure in the commercial environment of the companies.

These reasons left the designer with no time and/or no means for a thorough analysis. Thus, simple old analytical procedures are still used; simplifications are made. Such a rushed process forces the designers to add more layers, just to stay on the safe side. This process decreases the light-weight advantage of composite products.

The purpose of this article is to offer a new point of view towards a composite wing design method in terms of a comparison between analytical and numerical solutions of different beam shapes, ultimately evolved into a wing.

The process of a wing design will be illustrated on a real structure. The wing in question is that of a single seat, all composite glider plane, manufactured in the Kutná Hora, Czech Republic.

The results are evaluated using real laboratory tests (Juračka 2007; Matěják 2010) that took place at the Institute of Aerospace Engineering, Brno University of Technology.

\section{Problem}

The wing segment, which is being tested at the IAE, is of a full composite structure with thick carbon flanges. The first five tests were static tests, the next four (currently running the $9^{\text {th }}$ specimen test) were fatigue tests. Tests of two whole wings were carried out as well.

The simulation and calculation is based on the wing segment, as it is smaller, and more available test data exist.

The simulated test layout is shown in Figure 1.

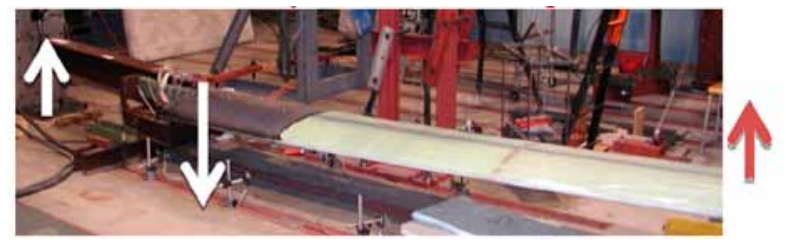

Fig. 1. Wing bend test

To find out where the problem of the premature failures may be, an in depth analysis of all the processes between the construction and structural design of the wing until the manufacturing is suggested.

This paper, however, focuses on the design stage only. It should answer the question as to what is the difference in the structural response of the wing segment to the introduced forces presented by analytical and numerical solutions.

This analysis will be performed on a step-evolution of the wing. It will start with a simple I-beam and finish with full wing geometry and all components of loading (Fig. 2).

To give credibility to this analysis, the finite element model shall be compared to the laboratory test.

During the laboratory tests, the failed component has been identified as the upper flange (Juračka 2007). Therefore, the analysis will focus solely on the upper flange in the root-rib area.

\section{Description of the 8 steps of evolution}

It has been already declared that the evolution will consist of eight steps. These step changes are to combine both, the geometry and loading schematics.

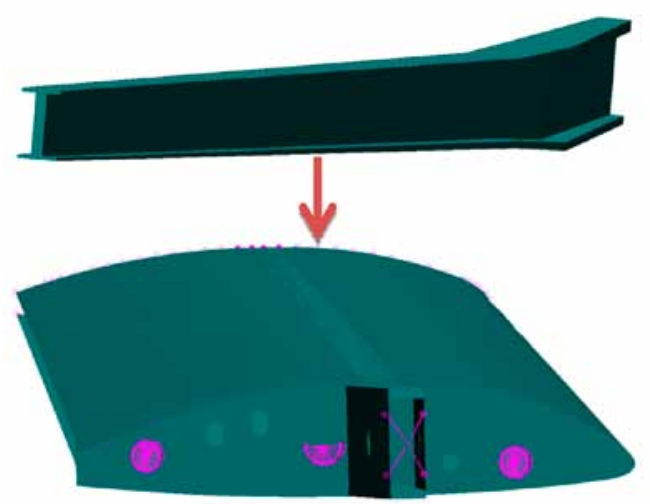

Fig. 2. Geometrical evolution: from an I-beam to a wing 
The change from the I-beam to the wing will be gradual. First, the inner part of the I-beam's (step\#1) cross-section will be replaced by a cantilever (step\#2). Instead of one web, there will be two; the cross-section is called the "box".

The next two steps are about the transition between the cantilever and the outer wing I-beam. These changes will increase the size of the transition wall into the ribwall (step\#3) and rib flanges (step\#4).

Then the rear spar and skin joins the construction (step\#5) and helps with the load re-distribution. The final geometrical change lies in adding small stiffening ribs and making holes (step\#6) in the root-rib (passages for the aileron and flap control mechanisms).

Further changes will take place during the loading. There will be three different loading steps, responding to the geometry change.

The first loading case (referred to as Loading $A$ ) is a simple three-point bending (Fig. 3) of an I-beam. This represents the fatigue laboratory test layout (Fig. 1). This loading is used for the geometrical configurations in step\#1-\#6.

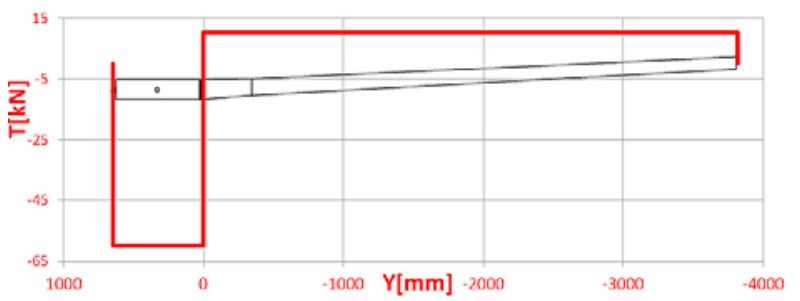

Fig. 3. Shear force distribution along the wing span, Loading A

The second loading scheme (referred to as Loading $B$ ) is linked to a geometry which includes the root rib and the skin with a rear spar. It will be combined with a full geometry of the wing and is called step\#7.

The introduction of this force represents the other wing. It does not affect the flanges, at least not from the first look, but it will be investigated, because this force significantly changes the shear force distribution in the root rib. The comparison of the shear force distribution in the root rib for Loading A and B is shown in Figure 4.

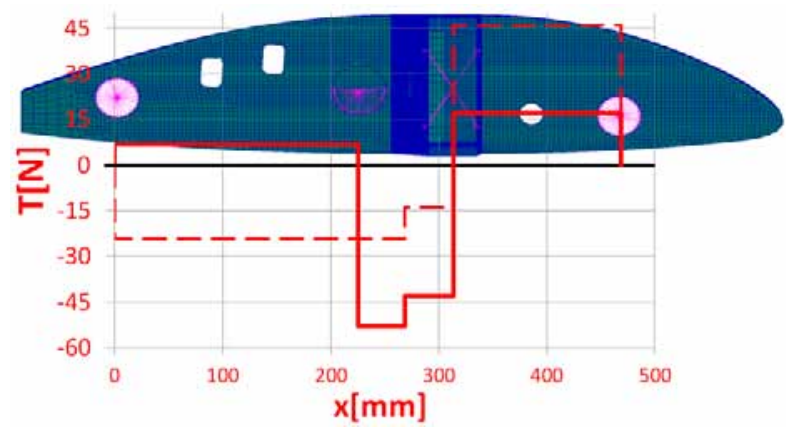

Fig. 4. Shear force distribution in the root rib: Loading $A$ and Loading $B$
Finally, Loading $C$ incorporates the rest of the aerodynamical forces: the twisting moment and the longitudinal-bending force (Fig. 5).

By definition, the beam will transfer only the forces $\mathrm{F}_{\mathrm{Z}}$ and $\mathrm{F}_{\mathrm{X}}$, whereas the skin will transfer the twisting moment. This assumption has been confirmed by three different finite element analyses where different combinations of forces and moments were evaluated with a resulting verdict that the twisting moment has no significant effect on the flanges or the beam itself.

In the light of this assumption the analytical calculation will not be taken into account at this moment.

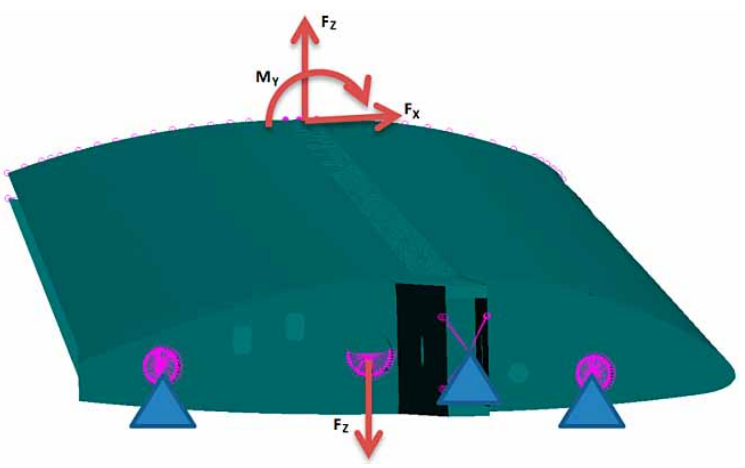

Fig. 5. Loading C

\section{Analytical solution}

The simple beam theory describes the stress state of a beam in terms of $\sigma_{1}$ and $\tau_{31}$. The normal stress $\sigma_{1}$ is pure tension or compression. The shear stress along the vertical axis inside the flange is a side-effect of the principal tension/compression stress:

$$
\begin{aligned}
\sigma_{1(Y, Z)} & =\frac{M O_{(Y)}}{J_{X}} \cdot Z ; \\
\tau_{31(Y, Z)} & =\frac{T_{(Y)} \cdot S_{(Y, Z)}}{B_{(Y)} \cdot J_{X(Y)}} .
\end{aligned}
$$

However, in the business practice, the approach that is usually applied to solve the stress state is a reduced Equation 1. It is simplified to calculate the average normal stress, whereas the shear stress is thought of as non-existing:

$$
\sigma_{1(Y)}=\frac{M O_{(Y)}}{H_{E F(Y)} \cdot A_{(Y)}} .
$$

The use of composite materials requires integrating all the plies into one global variable, such as $\mathrm{E}_{1}$. Furthermore, the stresses and deformations into the individual plies need to be recalculated.

The classical laminate theory is suitable (Liu 2003) for thin-wall structures, such as the skin of the wing. One of the assumptions during the theory development is that there are only planar forces and moments acing 
upon the laminate plate. This assumption can be illustrated by a vector of the loading forces and moments:

$$
\left[\begin{array}{l}
N \\
M
\end{array}\right]=\left[\begin{array}{llllll}
N_{X} & N_{Y} & N_{X Y} & M_{X} & M_{Y} & M_{X Y}
\end{array}\right]^{T} .
$$

This, however, may not apply for the flanges. The flanges are by no means thin and they aren't made of plies either.

\section{Numerical solution}

The numerical solution, or finite element analysis, is time consuming, expensive and demanding in terms of computers, software and engineering experience.

On the other hand, the FEE offers a very detailed analysis of all 6 stress components (for solid elements) and 3 stress components for shell elements, respectively.

It provides the possibility of incorporating the adhesive contact between parts. The loading can be much more complicated.

The MSC:Patran incorporates laminate modeller software for a simple definition of the plies. But it still uses similar assumptions as the CLT. Therefore, some important information about the stress is missing as well.

The flanges are ideal for applying solid HEX elements. This is beneficial, because it allows a direct and precise reading of the shear stress.

Another feature of the finite element analysis is the use of the contact function. This contact is a " $G$ "-type, $g$ stands for glue.

The layer of the glue that is in contact, instead of being equivalenced with the flange will significantly affect the stress in the interface.

\section{Validation of the FEM}

Since the laboratory tests not only provide the simple geometry and loading schematics, but also the results from the tests, it is possible to validate the finite element model against these laboratory measured parameters.

The finite element model has been validated with respect to the laboratory tests results (Matěják 2010). The validation parameters were the reaction forces, deflections and deformations. All these parameters were evaluated at $10 \%$ increments of the ultimate loading within the range from $10 \%$ to $60 \%$.

The typical differences are provided in Table 1.

Table 1. Validation of the FEA against the laboratory test.

\begin{tabular}{ll}
\hline \multicolumn{1}{c}{ Condition } & \multicolumn{1}{c}{ Difference [\%] } \\
\hline Reaction forces & $1.5-5.0$ \\
\hline Deflection & $10-15$ \\
\hline Deformation & 10 \\
\hline
\end{tabular}

Based on these results, the FE model is declared to be trustworthy, reflecting the real construction behavior and can be used for the evolution step analysis.

\section{Results of step evolution}

Based on the comparison of $\sigma_{1}$ stress in the upper flange, especially in the root section area, the following conclusions can be formulated:

- the transition between a single and double web spar creates a large concentration of stress (approximately twice as much of $\sigma_{1}$ stress in the root area);

- spreading supports from within the beam to the hinge points in the rib lowers the stress by $20 \%$;

- the flange of the rib has no significant effect on the peaks in the compression stress;

- the rear spar adds about $15 \%$ of tension stress to the root section of the flange;

- the introduction of a downward force decreases the compression stress peak at the root.

The previous Figures 6 and 7 demonstrated the relatively good agreement between the analytical and numerical solutions. Some effects that the analytical solution cannot take into account are visible, such as geometrical non-linearity.

One of the most important conditions that allows using the analytical equations states:

"The solution is valid only in a distance away of the areas of the boundary conditions." (Janíček et al. 1992).

That means that the analytical solution of our wing segment should not be valid, among other areas, at the root section.

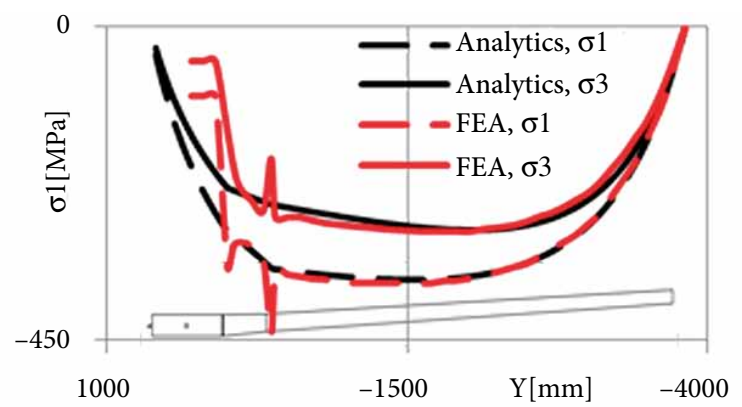

Fig. 6. Comparison of $\sigma_{1}$ solution, Loading A, Step 1

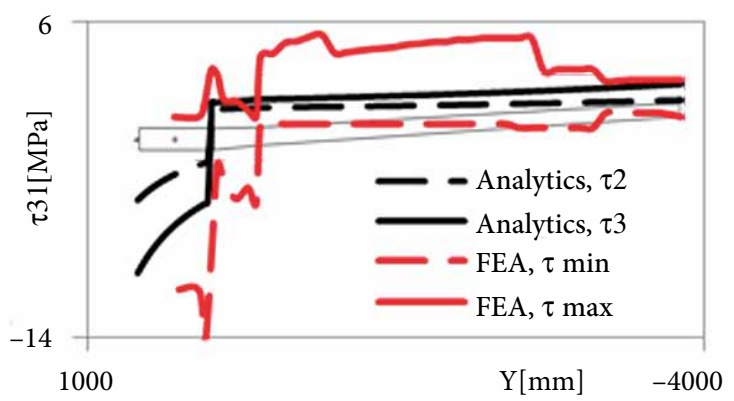

Fig. 7. Comparison of $\tau_{31}$ solution, Loading C, Step 8 
Figure 8 shows the $\sigma_{1}$ stresses obtained in the root area from both solutions of the upper flange for the Loading C:

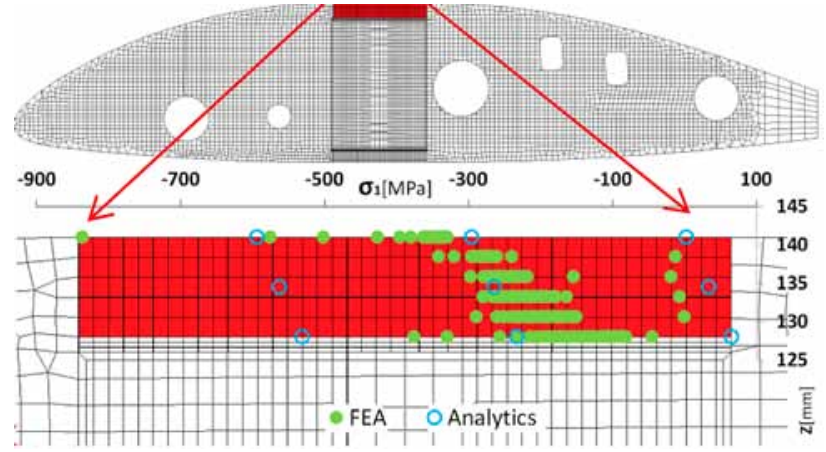

Fig. 8. Comparison of $\sigma_{1}$ solution, root, Loading $C$, Step 8

Figure 9 shows the influence of the web on the shear stress in the glue layer. This influence can be traces along the wing span.

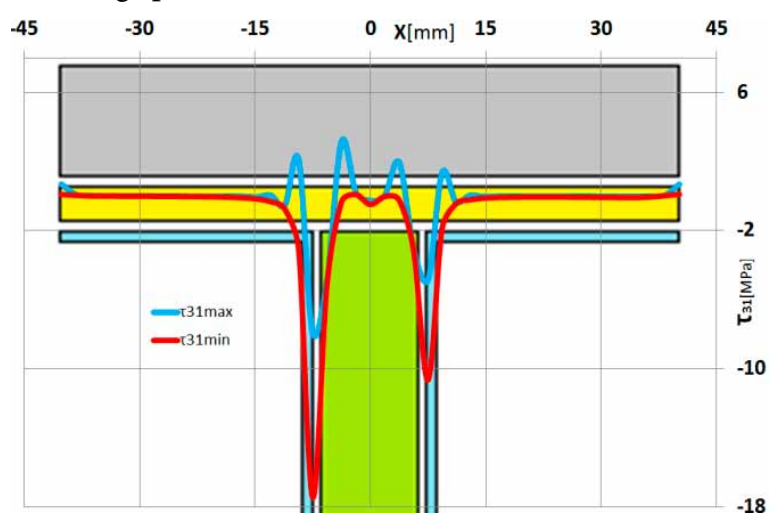

Fig. 9. Peaks of $\tau_{31}$ due to shear force in the glue layer

\section{Conclusions}

The step-evolution analysis has mapped the stress contribution of each geometrical change or change in the loading conditions.

Another outcome of the analysis is the comparison of the finite element analysis and the analytical solution.

The conclusion to be drawn from the method comparison is that there are significant differences, but neither is considered to be a severe overlook which might cause the failures of the structure.

The biggest impact of this in depth analysis is the definition of new questions to be answered. The questions concern the stress interaction.

The wing construction and loading strongly resemble the three-point bending tests. This obvious analogy evokes the suspicion that the elliptical failure envelope, known from the three-point bend tests, may in some way apply even here, in the wing construction, and explain the premature failures.
The shear stress $\tau_{31}$ is caused by the shear force. Focusing on the shear force distribution between the components, a distinct pattern appears in the glue layer between the web and the flange.

The peak stresses are located in the positions of the glass-fibre walls of the web.

\section{Acknowledgements}

These outputs were supported by the project KhAI-ERA Integrating the National Aerospace University KhAIERA into ERA (FP7-INCO-2011-6) with the cooperation of NETME Centre, regional R\&D centre built with the financial support from the Operational Programme Research and Development for Innovations within the project NETME Centre (New Technologies for Mechanical Engineering), Reg. No. CZ.1.05/2.1.00/01.0002.

\section{References}

Juračka, J. 2007. Referenční statická zkouška segmentu křídla. IAE report. $34 \mathrm{p}$.

Janíček, P.; Ondráček, E.; Vrbka, J. 1992. Pružnost a pevnost I. VUT v Brně.

Liu, Y. 2003. Introduction to the finite element method. CAE Research Laboratory. $188 \mathrm{p}$.

Matěják, V. 2010. Laboratory strength test methodology of $304 \mathrm{~s}$ wing. IAE report. $13 \mathrm{p}$. 